\title{
Multiple Positive Solutions for a Class of $m$-Point Boundary Value Problems on Time Scales
}

\author{
Meiqiang Feng, ${ }^{1}$ Xuemei Zhang, ${ }^{2,3}$ and Weigao $\mathrm{Ge}^{3}$ \\ ${ }^{1}$ School of Science, Beijing Information Science \& Technology University, \\ Beijing 100192, China \\ ${ }^{2}$ Department of Mathematics and Physics, North China Electric Power University, \\ Beijing 102206, China \\ ${ }^{3}$ Department of Applied Mathematics, Beijing Institute of Technology, \\ Beijing 100081, China
}

Correspondence should be addressed to Xuemei Zhang, zxm74@sina.com

Received 1 December 2008; Revised 15 April 2009; Accepted 10 June 2009

Recommended by Victoria Otero-Espinar

By constructing an available integral operator and combining Krasnosel'skii-Zabreiko fixed point theorem with properties of Green's function, this paper shows the existence of multiple positive solutions for a class of $m$-point second-order Sturm-Liouville-like boundary value problems on time scales with polynomial nonlinearity. The results significantly extend and improve many known results for both the continuous case and more general time scales. We illustrate our results by one example, which cannot be handled using the existing results.

Copyright (c) 2009 Meiqiang Feng et al. This is an open access article distributed under the Creative Commons Attribution License, which permits unrestricted use, distribution, and reproduction in any medium, provided the original work is properly cited.

\section{Introduction}

Recently, there have been many papers working on the existence of positive solutions to boundary value problems for differential equations on time scales; see, for example, [120]. This has been mainly due to its unification of the theory of differential and difference equations. An introduction to this unification is given in [11, 12, 18, 19]. Now, this study is still a new area of fairly theoretical exploration in mathematics. However, it has led to several important applications, for example, in the study of insect population models, neural networks, heat transfer, and epidemic models; see, for example, [10, 11]. For some other excellent results and applications of the case that boundary value problems on time scales to a variety of problems from Khan et al. [21], Agarose et al. [22], Wang [23], Sun [24], Feng et al. [25], Feng et al. [26] and Feng et al. [27].

Motivated by the works mentioned above, we intend in this paper to study the existence of multiple positive solutions for the second-order $m$-point nonlinear dynamic 
equation on time scales with polynomial nonlinearity:

$$
\begin{gathered}
-\left[p(t) x^{\nabla}\right]^{\Delta}(t)+q(t) x(t)=f(t, x(t)), \quad t_{1}<t<t_{m}, \\
\alpha x\left(t_{1}\right)-\beta p\left(t_{1}\right) x^{\nabla}\left(t_{1}\right)=\sum_{i=2}^{m-1} a_{i} x\left(t_{i}\right), \\
\gamma x\left(t_{m}\right)+\delta p\left(t_{m}\right) x^{\nabla}\left(t_{m}\right)=\sum_{i=2}^{m-1} b_{i} x\left(t_{i}\right),
\end{gathered}
$$

where $\mathbb{T}$ is a time scale,

$$
p, q:\left[t_{1}, t_{m}\right] \longrightarrow(0, \infty), \quad p \in C^{\Delta}\left[t_{1}, t_{m}\right), q \in C\left[t_{1}, t_{m}\right] ;
$$

the points $t_{i} \in \mathbb{T}_{k}^{k}$ for $i \in\{1,2, \ldots, m\}$ with $t_{1}<t_{2}<\cdots<t_{m}$

$$
\begin{aligned}
& \alpha, \gamma, \beta, \delta \in[0, \infty) \quad \alpha \gamma+\alpha \delta+\beta \gamma>0, \quad a_{i}, b_{i} \in[0, \infty), i \in\{2,3, \ldots, m-1\} ; \\
& f(t, x)=\sum_{j=1}^{n} c_{j}(t) x^{v_{j}}, \quad c_{j} \in C\left(\left[t_{1}, t_{m}\right],[0,+\infty)\right), v_{j} \in[0, \infty), j=1,2, \ldots, n .
\end{aligned}
$$

Recently, $\mathrm{Xu}$ [28] considered the following second-order two-point impulsive singular differential equations boundary value problem:

$$
\begin{aligned}
y^{\prime \prime}+\sum_{j=1}^{n} a_{j}(t) x^{\alpha_{j}} & =0, \quad 0<t<1, \quad t \neq t_{1}, \\
\left.\Delta y\right|_{t=t_{1}} & =I\left(y\left(t_{1}\right)\right), \\
y(0) & =y(1)=0 .
\end{aligned}
$$

By means of fixed point index theory in a cone, the author established the existence of two nonnegative solutions for problem (1.5).

More recently, by applying Guo-Krasnosel'skii fixed point theorem in a cone, Anderson and Ma [6] established the existence of at least one positive solution to the multipoint time-scale eigenvalue problem:

$$
\begin{gathered}
{\left[p y^{\nabla}\right]^{\Delta}(t)-q(t) y(t)+\lambda h(t) f(y)=0, \quad t_{1}<t<t_{n}} \\
\alpha y\left(t_{1}\right)-\beta p\left(t_{1}\right) y^{\nabla}\left(t_{1}\right)=\sum_{i=2}^{n-1} a_{i} y\left(t_{i}\right), \\
\gamma x\left(t_{n}\right)+\delta p\left(t_{n}\right) x^{\nabla}\left(t_{n}\right)=\sum_{i=2}^{n-1} b_{i} y\left(t_{i}\right),
\end{gathered}
$$

where $f:[0, \infty) \rightarrow[0, \infty)$ is continuous. 
As far as we know, there is no paper to study the existence of multiple positive solutions to problem (1.1) on time scales with polynomial nonlinearity. The objective of the present paper is to fill this gap. On the other hand, many difficulties occur when we study BVPs on time scales. For example, basic tools from calculus such as Fermat's theorem, Rolle's theorem and the intermediate value theorem may not necessarily hold. So it is interesting and important to discuss the problem (1.1). The purpose of this paper is to prove that the problem (1.1) possesses at least two positive solutions. Moreover, the methods used in this paper are different from $[6,28]$ and the results obtained in this paper generalize some results in $[6,28]$ to some degree.

The time scale related notations adopted in this paper can be found, if not explained specifically, in almost all literature related to time scales. The readers who are unfamiliar with this area can consult for example [11, 12, 18, 19] for details.

For convenience, we list the following well-known definitions.

Definition 1.1. A time scale $\mathbb{T}$ is a nonempty closed subset of $\mathbb{R}$.

Definition 1.2. Define the forward (backward) jump operator $\sigma(t)$ at $t$ for $t<\sup \mathbb{T}(\rho(t)$ at $t$ for $t>\inf \mathbb{T})$ by $\sigma(t)=\inf \{\tau>t: \tau \in \mathbb{T}\} \quad(\rho(t)=\sup \{\tau<t: \tau \in \mathbb{T}\})$ for all $t \in \mathbb{T}$.

We assume throughout that $\mathbb{T}$ has the topology that it inherits from the standard topology on $\mathbb{R}$ and say $t$ is right-scattered, left-scattered, right-dense and left-dense if $\sigma(t)>$ $t, \rho(t)<t, \sigma(t)=t$ and $\rho(t)=t$, respectively. Finally, we introduce the sets $\mathbb{T}^{k}$ and $\mathbb{T}_{k}$. which are derived from the time scale $\mathbb{T}$ as follows. If $\mathbb{T}$ has a left-scattered maximum $t_{1}^{*}$, then $\mathbb{T}^{k}=\mathbb{T}-t_{1}^{*}$, otherwise $\mathbb{T}^{k}=\mathbb{T}$. If $\mathbb{T}$ has a right-scattered minimum $t_{2}^{*}$, then $\mathbb{T}_{k}=\mathbb{T}-t_{2}^{*}$, otherwise $\mathbb{T}_{k}=\mathbb{T}$.

Definition 1.3. Fix $t \in \mathbb{T}$ and let $y: \mathbb{T} \rightarrow \mathbb{R}$. Define $y^{\Delta}(t)$ to be the number (if it exists) with the property that given $\varepsilon>0$ there is a neighborhood $U$ of $t$ with

$$
\left|[y(\sigma(t))-y(s)]-y^{\Delta}(t)[\sigma(t)-s]\right|<\varepsilon|\sigma(t)-s|
$$

for all $s \in U$, where $y^{\Delta}$ denotes the (delta) derivative of $y$ with respect to the first variable, then

$$
g(t):=\int_{a}^{t} \omega(t, \tau) \Delta \tau
$$

implies

$$
g^{\Delta}(t)=\int_{a}^{t} \omega^{\Delta}(t, \tau) \Delta \tau+\omega(\sigma(t), \tau)
$$

Definition 1.4. Fix $t \in \mathbb{T}$ and let $y: \mathbb{T} \rightarrow \mathbb{R}$. Define $y^{\nabla}(t)$ to be the number (if it exists) with the property that given $\varepsilon>0$ there is a neighborhood $U$ of $t$ with

$$
\left|[y(\rho(t))-y(s)]-y^{\nabla}(t)[\rho(t)-s]\right|<\varepsilon|\rho(t)-s|
$$

for all $s \in U$. Call $y^{\nabla}(t)$ the nabla derivative of $y(t)$ at the point $t$. 
If $\mathbb{T}=\mathbb{R}$ then $f^{\Delta}(t)=f^{\nabla}(t)=f^{\prime}(t)$. If $\mathbb{T}=\mathbb{Z}$ then $f^{\Delta}(t)=f(t+1)-f(t)$ is the forward difference operator while $f^{\nabla}(t)=f(t)-f(t-1)$ is the backward difference operator.

Definition 1.5. A function $f: \mathbb{T} \rightarrow \mathbb{R}$ is called rd-continuous provided it is continuous at all right dense points of $\mathbb{T}$ and its left sided limit exists (finite) at left dense points of $\mathbb{T}$. We let $C_{\text {rd }}^{0}(\mathbb{T})$ denote the set of rd-continuous functions $f: \mathbb{T} \rightarrow \mathbb{R}$.

Definition 1.6. A function $f: \mathbb{T} \rightarrow \mathbb{R}$ is called ld-continuous provided it is continuous at all left dense points of $\mathbb{T}$ and its right sided limit exists (finite) at right dense points of $\mathbb{T}$. We let $C_{\mathrm{ld}}^{0}(\mathbb{T})$ denote the set of ld-continuous functions $f: \mathbb{T} \rightarrow \mathbb{R}$.

Definition 1.7. A function $F: \mathbb{T}^{k} \rightarrow \mathbb{R}$ is called a delta-antiderivative of $f: \mathbb{T}^{k} \rightarrow \mathbb{R}$ provided $F^{\Delta}(t)=f(t)$ holds for all $t \in \mathbb{T}^{k}$. In this case we define the delta integral of $f$ by

$$
\int_{a}^{t} f(s) \Delta s=F(t)-F(a)
$$

for all $a, t \in \mathbb{T}$.

Definition 1.8. A function $\Phi: \mathbb{T}_{k} \rightarrow \mathbb{R}$ is called a nabla-antiderivative of $f: \mathbb{T}_{k} \rightarrow \mathbb{R}$ provided $\Phi^{\nabla}(t)=f(t)$ holds for all $t \in \mathbb{T}_{k}$. In this case we define the nabla integral of $f$ by

$$
\int_{a}^{t} f(s) \nabla s=\Phi(t)-\Phi(a)
$$

for all $a, t \in \mathbb{T}$.

\section{Preliminaries}

In this section, we provide some necessary background. In particular, we state some properties of Green's function associated with problem (1.1), and we then state a fixed-point theorem which is crucial to prove our main results.

The basic space used in this paper is $E=C\left[\rho\left(t_{1}\right), t_{m}\right]$. It is well known that $E$ is a Banach space with the norm $\|\cdot\|$ defined by $\|x\|=\sup _{t \in\left[\rho\left(t_{1}\right), t_{m}\right]}|x(t)|$. Let $P$ be a cone of $E$, $P_{r}=\{x \in P:\|x\| \leq r\}, \partial P_{r}=\{x \in P:\|x\|=r\}$, where $r>0$. by

In this paper, the Green's function of the corresponding homogeneous BVP is defined

$$
G(t, s)=\frac{1}{d} \begin{cases}\psi(t) \phi(s), & \text { if } \rho\left(t_{1}\right) \leq t \leq s \leq t_{m} \\ \psi(s) \phi(t), & \text { if } \rho\left(t_{1}\right) \leq s \leq t \leq t_{m}\end{cases}
$$

where

$$
d:=\alpha \phi\left(t_{1}\right)-\beta p\left(t_{1}\right) \phi^{\nabla}\left(t_{1}\right)=\gamma \psi\left(t_{m}\right)+\delta p\left(t_{m}\right) \psi^{\nabla}\left(t_{m}\right),
$$


and $\phi$ and $\psi$ satisfy

$$
\begin{array}{ccc}
-\left(p \psi^{\nabla}\right)^{\Delta}(t)+q(t) \psi(t)=0, & \psi\left(t_{1}\right)=\beta, & p\left(t_{1}\right) \psi^{\nabla}\left(t_{1}\right)=\alpha, \\
-\left(p \phi^{\nabla}\right)^{\Delta}(t)+q(t) \phi(t)=0, & \phi\left(t_{m}\right)=\delta, & p\left(t_{m}\right) \phi^{\nabla}\left(t_{m}\right)=-\gamma,
\end{array}
$$

respectively.

Lemma 2.1 (see [6]). Assume that (1.2) and (1.3) hold. Then $d>0$ and the functions $\psi$ and $\phi$ satisfy

$$
\begin{gathered}
\psi(t) \geq 0, \quad t \in\left[\rho\left(t_{1}\right), t_{m}\right], \quad \psi(t)>0, \quad t \in\left(\rho\left(t_{1}\right), t_{m}\right], \\
p(t) \psi^{\nabla}(t) \geq 0, \quad t \in\left[\rho\left(t_{1}\right), t_{m}\right], \quad \phi(t) \geq 0, \quad t \in\left[\rho\left(t_{1}\right), t_{m}\right], \\
\phi(t)>0, \quad t \in\left[\rho\left(t_{1}\right), t_{m}\right), \quad p(t) \phi^{\nabla}(t) \leq 0, \quad t \in\left[\rho\left(t_{1}\right), t_{m}\right] .
\end{gathered}
$$

From Lemma 2.1 and the definition of $G(t, s)$, we can prove that $G(t, s)$ has the following properties.

Proposition 2.2. For $t, s \in\left[\xi_{1}, \xi_{2}\right]$, one has

$$
G(t, s)>0,
$$

where $\xi_{1}, \xi_{2} \in \mathbb{T}_{k}^{k}, \rho\left(t_{1}\right)<\xi_{1}<\xi_{2}<t_{m}$. holds.

In fact, from Lemma 2.1, we have $\psi(t)>0, \quad \phi(t)>0$ for $t \in\left[\xi_{1}, \xi_{2}\right]$. Therefore (2.5)

Proposition 2.3. If (1.2) holds, then for $t, s \in\left[\rho\left(t_{1}\right), t_{m}\right] \times\left[\rho\left(t_{1}\right), t_{m}\right]$, one has

$$
0 \leq G(t, s) \leq G(s, s)
$$

Proof. In fact, from Lemma 2.1, we obtain $\psi(t) \geq 0, \phi(t) \geq 0$ for $t \in\left[\rho\left(t_{1}\right), t_{m}\right]$. So $G(t, s) \geq 0$.

On the other hand, from Lemma 2.1, we know that $p(t) \psi^{\nabla}(t) \geq 0, p(t) \phi^{\nabla}(t) \leq 0$ for $t \in\left[\rho\left(t_{1}\right), t_{m}\right]$. This together with $p(t)>0$ implies that $\psi^{\nabla}(t) \geq 0, \phi^{\nabla}(t) \leq 0$ for $t \in\left[\rho\left(t_{1}\right), t_{m}\right]$. Hence $\psi(t)$ is nondecreasing on $\left[\rho\left(t_{1}\right), t_{m}\right], \phi$ is nonincreasing on $\left[\rho\left(t_{1}\right), t_{m}\right]$. So $(2.6)$ holds.

Proposition 2.4. For all $t \in\left[\xi_{1}, \xi_{2}\right], s \in\left[\rho\left(t_{1}\right), t_{m}\right]$ one has

$$
G(t, s) \geq \sigma(t) G(s, s)
$$


where

$$
\sigma(t):=\min \left\{\frac{\psi(t)}{\psi\left(t_{m}\right)}, \frac{\phi(t)}{\phi\left(\rho\left(t_{1}\right)\right)}\right\}
$$

Proof. In fact, for $t \in\left[\xi_{1}, \xi_{2}\right]$, we have

$$
\frac{G(t, s)}{G(s, s)} \geq \min \left\{\frac{\psi(t)}{\psi(s)}, \frac{\phi(t)}{\phi(s)}\right\} \geq \min \left\{\frac{\psi(t)}{\psi\left(t_{m}\right)}, \frac{\phi(t)}{\phi\left(\rho\left(t_{1}\right)\right)}\right\}=: \sigma(t) .
$$

Therefore (2.7) holds.

It is easy to see that $0<\sigma(t)<1$, for $t \in\left[\xi_{1}, \xi_{2}\right]$. Thus, there exists $\bar{\gamma}>0$ such that $G(t, s) \geq \bar{\gamma} G(s, s)$ for $t \in\left[\xi_{1}, \xi_{2}\right]$, where

$$
\bar{\gamma}=\min \left\{\sigma(t): t \in\left[\xi_{1}, \xi_{2}\right]\right\}
$$

We remark that Proposition 2.2 implies that there exists $\tau>0$ such that for $t, s \in\left[\xi_{1}, \xi_{2}\right]$

$$
G(t, s) \geq \tau
$$

Set

$$
D:=\left|\begin{array}{cc}
-\sum_{i=1}^{m-2} a_{i} \psi\left(t_{i}\right) & d-\sum_{i=1}^{m-2} a_{i} \phi\left(t_{i}\right) \\
d-\sum_{i=1}^{m-2} b_{i} \psi\left(t_{i}\right) & -\sum_{i=1}^{m-2} b_{i} \phi\left(t_{i}\right)
\end{array}\right|
$$

Lemma 2.5 (see [6]). Assume that (1.2) and (1.3) hold. If $D \neq 0$ and $u \in C_{r d}\left[t_{1}, t_{m}\right]$, then the nonhomogeneous boundary value problem

$$
\begin{gathered}
-\left[p(t) x^{\nabla}\right]^{\Delta}(t)+q(t) x(t)=u(t), \quad t_{1}<t<t_{m}, \\
\alpha x\left(t_{1}\right)-\beta p\left(t_{1}\right) x^{\nabla}\left(t_{1}\right)=\sum_{i=2}^{m-1} a_{i} x\left(t_{i}\right) \\
\gamma x\left(t_{m}\right)+\delta p\left(t_{m}\right) x^{\nabla}\left(t_{m}\right)=\sum_{i=2}^{m-1} b_{i} x\left(t_{i}\right)
\end{gathered}
$$

has a unique solution $x$ for which the formula

$$
x(t)=\int_{t_{1}}^{t_{m}} G(t, s) u(s) \Delta s+\Gamma(u(t)) \psi(t)+\Upsilon(u(t)) \phi(t)
$$


holds, where

$$
\begin{gathered}
\Gamma(u(s)):=\frac{1}{D}\left|\begin{array}{ll}
\sum_{i=2}^{m-1} a_{i} \int_{t_{1}}^{t_{m}} G\left(t_{i}, s\right) u(s) \Delta s & d-\sum_{i=2}^{m-1} a_{i} \phi\left(t_{i}\right) \\
\sum_{i=2}^{m-1} b_{i} \int_{t_{1}}^{t_{m}} G\left(t_{i}, s\right) u(s) \Delta s & -\sum_{i=2}^{m-1} b_{i} \phi\left(t_{i}\right)
\end{array}\right|, \\
\Upsilon(u(s)):=\frac{1}{D}\left|\begin{array}{cc}
-\sum_{i=2}^{m-1} a_{i} \psi\left(t_{i}\right) & \sum_{i=2}^{m-1} a_{i} \int_{t_{1}}^{t_{m}} G\left(t_{i}, s\right) u(s) \Delta s \\
d-\sum_{i=2}^{m-1} b_{i} \psi\left(\xi_{i}\right) & \sum_{i=2}^{m-1} b_{i} \int_{t_{1}}^{t_{m}} G\left(t_{i}, s\right) u(s) \Delta s
\end{array}\right| .
\end{gathered}
$$

By similar method, one can define

$$
\begin{array}{llll}
\Gamma_{0}\left(f\left(t, x_{0}(t)\right)\right), & \Gamma_{1}\left(f\left(t, x_{1}(t)\right)\right), & \Gamma_{2}\left(f\left(t, x_{2}(t)\right)\right), & \Gamma_{*}\left(f\left(t, x_{*}(t)\right)\right), \\
\Upsilon_{0}\left(f\left(t, x_{0}(t)\right)\right), & \Upsilon_{1}\left(f\left(t, x_{1}(t)\right)\right), & \Upsilon_{2}\left(f\left(t, x_{2}(t)\right)\right), & \Upsilon_{*}\left(f\left(t, x_{*}(t)\right)\right) .
\end{array}
$$

The following lemma is crucial to prove our main results.

Lemma 2.6 (see[29, 30]). Let $\Omega_{1}$ and $\Omega_{2}$ be two bounded open sets in a real Banach space $E$, such that $0 \in \Omega_{1}$ and $\bar{\Omega}_{1} \subset \Omega_{2}$. Let the operator $A: P \cap\left(\bar{\Omega}_{2} \backslash \Omega_{1}\right) \rightarrow P$ be completely continuous, where $P$ is a cone in $E$. Suppose that one of the two conditions

$$
\text { (i) } \quad A x \nsupseteq x, \quad \forall x \in P \cap \partial \Omega_{1} ; \quad A x \not x, \quad \forall x \in P \cap \partial \Omega_{2},
$$

or

$$
\text { (ii) } \quad A x \notin x, \quad \forall x \in P \cap \partial \Omega_{1} ; \quad A x \geq x, \quad \forall x \in P \cap \partial \Omega_{2} \text {, }
$$

is satisfied. Then $A$ has at least one fixed point in $P \cap\left(\Omega_{2} \backslash \bar{\Omega}_{1}\right)$.

\section{Main Results}

In this section, we apply Lemma 2.6 to establish the existence of at least two positive solutions for BVP (1.1).

The following assumptions will stand throughout this paper.

$\left(H_{1}\right)$ There exist $v_{j 1}<1, v_{j 2}>1$ such that

$$
\inf _{t \in\left[\xi_{1}, \xi_{2}\right]} c_{j 1}(t)=\tau_{1}>0, \quad \inf _{t \in\left[\xi_{1}, \xi_{2}\right]} c_{j 2}(t)=\tau_{2}>0, \quad j=1,2, \ldots, n,
$$

where $v_{j 1}, v_{j 2}, c_{j 1}(t)$ and $c_{j 2}(t)$ are defined in (1.4), respectively. 
$\left(H_{2}\right)$ We have

$$
D<0, \quad d-\sum_{i=2}^{m-1} a_{i} \phi\left(t_{i}\right)>0, \quad d-\sum_{i=2}^{m-1} b_{i} \psi\left(t_{i}\right)>0
$$

for $d$ and $D$ given in (2.2) and (2.12), respectively. properties.

If $\left(H_{2}\right)$ holds, then we can show that $\Gamma(f(t, x)), \Upsilon(f(t, x))$ have the following

Proposition 3.1. If (1.2)-(1.4) and $\left(H_{2}\right)$ hold, then from (2.15), for $x \in C\left[\rho\left(t_{1}\right), t_{m}\right]$, one has

$$
|\Gamma(f(t, x))| \leq \frac{1}{D}\left|\begin{array}{|cc}
\sum_{i=2}^{m-1} a_{i} & d-\sum_{i=2}^{m-1} a_{i} \phi\left(t_{i}\right) \\
\sum_{i=2}^{m-1} b_{i} & -\sum_{i=2}^{m-1} b_{i} \phi\left(t_{i}\right)
\end{array}\right| M \sum_{j=1}^{n}\left\|c_{j}\right\|_{L}\|x\|^{v_{j}}=: \widetilde{\Gamma} M \sum_{j=1}^{n}\left\|c_{j}\right\|_{L}\|x\|^{v_{j}}
$$

where $\left\|c_{j}\right\|_{L}:=\int_{t_{1}}^{t_{m}}\left|c_{j}(s)\right| \Delta s, M=\max _{(t, s) \in\left[\rho\left(t_{1}\right), t_{m}\right] \times\left[\rho\left(t_{1}\right), t_{m}\right]} G(t, s)$.

Proof. Let

$$
\begin{array}{ll}
G=\sum_{i=2}^{m-1} a_{i} \int_{t_{1}}^{t_{m}} G\left(t_{i}, s\right) f(s, x(s)) \Delta s, \quad H=d-\sum_{i=2}^{m-1} a_{i} \phi\left(t_{i}\right), \\
F=\sum_{i=2}^{m-1} b_{i} \int_{t_{1}}^{t_{m}} G\left(t_{i}, s\right) f(s, x(s)) \Delta s, \quad Q=-\sum_{i=2}^{m-1} b_{i} \phi\left(t_{i}\right) .
\end{array}
$$

Then from (1.2)-(1.4) and $\left(H_{2}\right)$, we obtain $G \geq 0, F \geq 0, H>0, Q \leq 0$. Therefore, $G Q \leq$ $0,-F H \leq 0$.

On the other hand, since

$$
\int_{t_{1}}^{t_{m}} G\left(t_{i}, s\right) f(s, x(s)) \Delta s \leq M \sum_{j=1}^{n}\left\|c_{j}\right\|_{L}\|x\|^{v_{j}}=: \Lambda,
$$

we have $G \leq \sum_{i=2}^{m-1} a_{i} \Lambda, F \leq \sum_{i=2}^{m-1} b_{i} \Lambda$. So one has

$$
\sum_{i=2}^{m-1} a_{i} \Lambda Q-H \sum_{i=2}^{m-1} b_{i} \Lambda \leq G Q-F H \leq 0
$$

This and $D<0$ imply (3.3) holds. 
Proposition 3.2. If (1.2)-(1.4) and $\left(H_{2}\right)$ hold, then from (2.16), $x \in C\left[\rho\left(t_{1}\right), t_{m}\right]$, one has

$$
|\Upsilon(f(t, x))| \leq \frac{1}{D}\left|\begin{array}{cc}
-\sum_{i=2}^{m-1} a_{i} \psi\left(t_{i}\right) & \sum_{i=2}^{m-1} a_{i} \\
d-\sum_{i=2}^{m-1} b_{i} \psi\left(\xi_{i}\right) & \sum_{i=2}^{m-1} b_{i}
\end{array}\right| M \sum_{j=1}^{n}\left\|c_{j}\right\|_{L}\|x\|^{v_{j}}=: \tilde{\Upsilon} M \sum_{j=1}^{n}\left\|c_{j}\right\|_{L}\|x\|^{v_{j}}
$$

Proof. The proof is similar to that of Proposition 3.1. So we omit it.

For the sake of applying fixed point theorem on cone, we construct a cone in $E=$ $C\left[\rho\left(t_{1}\right), t_{m}\right]$ by

$$
P=\left\{x \in E: x(t) \geq 0, t \in\left[\rho\left(t_{1}\right), t_{m}\right], \min _{t \in\left[\xi_{1}, \xi_{2}\right]} x(t) \geq \bar{\gamma}\|x\|\right\}
$$

where $\bar{\gamma}$ is defined in (2.10).

Define $A: P \rightarrow P$ by

$$
(A x)(t)=\int_{t_{1}}^{t_{m}} G(t, s) f(s, x(s)) \Delta s+\Gamma(f(t, x(t))) \psi(t)+\Upsilon(f(t, x(t))) \phi(t) .
$$

By (2.14), it is well known that the problem (1.1) has a positive solution $x$ if and only if $x \in P$ is a fixed point of $A$.

Lemma 3.3. Suppose that (1.2)-(1.4) and $\left(H_{1}\right)-\left(H_{2}\right)$ hold. Then $A(P) \subset P$ and $A: P \rightarrow P$ is completely continuous.

Proof. For $x \in P$, by (2.14), we have $A x(t) \geq 0$ and

$$
\|A x\| \leq \int_{t_{1}}^{t_{m}} G(s, s) f(s, x(s)) \Delta s+\Gamma(f(t, x(t))) \psi\left(t_{m}\right)+\Upsilon(f(t, x(t))) \phi\left(\rho\left(t_{1}\right)\right) .
$$

On the other hand, for $t \in\left[\xi_{1}, \xi_{2}\right]$, by (3.9),(3.10) and (2.7), we obtain

$$
\begin{aligned}
\min _{t \in\left[\xi_{1}, \xi_{2}\right]} A x(t) & =\min _{t \in\left[\xi_{1}, \xi_{2}\right]}\left[\int_{t_{1}}^{t_{m}} G(t, s) f(s, x(s)) \Delta s+\Gamma(f(t, x(t))) \psi(t)+\Upsilon(f(t, x(t))) \phi(t)\right] \\
& \geq \sigma(t)\left[\int_{t_{1}}^{t_{m}} G(s, s) f(s, x(s)) \Delta s+\Gamma(f(t, x(t))) \psi\left(t_{m}\right)+\Upsilon(f(t, x(t))) \phi\left(\rho\left(t_{1}\right)\right)\right] \\
& \geq \sigma(t)\|A x\| \geq \bar{\gamma}\|A x\| .
\end{aligned}
$$

Therefore $A x \in P$, that is, $A(P) \subset P$.

Next by standard methods and the Ascoli-Arzela theorem one can prove that $A: P \rightarrow$ $P$ is completely continuous. So it is omitted. 
Theorem 3.4. Suppose that (1.2)-(1.4) and $\left(H_{1}\right)-\left(H_{2}\right)$ hold. Then problem (1.1) has at least two positive solutions provided

$$
\sum_{j=1}^{n}\left\|c_{j}\right\|_{L}\left[1+\tilde{\Gamma} \psi\left(t_{n}\right)+\tilde{\Upsilon} \phi\left(t_{1}\right)\right]<M^{-1}
$$

where $\widetilde{\Gamma}, \tilde{\Upsilon}$ and $M$ are defined in (3.3), (3.7) and in Proposition 3.1, respectively.

Proof. Let $A$ be the cone preserving, completely continuous operator that was defined by (3.9).

Let $S_{l}=\{x \in E:\|x\|<l\}$, where $l>0$. Choosing $r$ and $\bar{r}$ satisfy

$$
\begin{aligned}
& 0<r<\min \left\{1,\left(\tau \tau_{1}\left(\xi_{2}-\xi_{1}\right)\right)^{1 /\left(1-v_{j 1}\right)} \bar{\gamma}^{v_{j 1} /\left(1-v_{j 1}\right)}\right\} \\
& \bar{r}>\max \left\{1,\left(\tau \tau_{2}\left(\xi_{2}-\xi_{1}\right)\right)^{-1 /\left(v_{j 2}-1\right)} \bar{\gamma}^{\left(-v_{j 2}\right) /\left(v_{j 2}-1\right)}\right\}
\end{aligned}
$$

Now we prove that

$$
\begin{aligned}
& A x \not x, \quad \forall x \in P \cap \partial S_{r}, \\
& A x \not \leq x, \quad \forall x \in P \cap \partial S_{\bar{r}} .
\end{aligned}
$$

In fact, if there exists $x_{1} \in P \cap \partial S_{r}$ such that $A x_{1} \leq x_{1}$, then for $t \in\left[\xi_{1}, \xi_{2}\right]$, we have

$$
\begin{aligned}
x_{1}(t) & \geq A x_{1}(t) \\
& =\int_{t_{1}}^{t_{m}} G(t, s) f\left(s, x_{1}(s)\right) \Delta s+\Gamma_{1}\left(f\left(t, x_{1}(t)\right)\right) \psi(t)+\Upsilon_{1}\left(f\left(t, x_{1}(t)\right)\right) \phi(t) \\
& \geq \int_{t_{1}}^{t_{m}} G(t, s) f\left(s, x_{1}(s)\right) \Delta s \\
& \geq \int_{\xi_{1}}^{\xi_{2}} G(t, s) c_{j 1}(s)\left[x_{1}(s)\right]^{v_{j 1}} \Delta s \\
& \geq \tau \tau_{1}\left(\xi_{2}-\xi_{1}\right) \bar{\gamma}^{v_{j 1}}\left\|x_{1}\right\|^{v_{j 1}},
\end{aligned}
$$

where $\Gamma_{1}(f(t, x(t))), \Upsilon_{1}(f(t, x(t)))$ defined by (2.17).

Therefore $r \geq \tau \tau_{1}\left(\xi_{2}-\xi_{1}\right) \bar{\gamma}^{v_{j 1}} r^{v_{j 1}}$, that is, $r \geq\left(\tau \tau_{1}\left(\xi_{2}-\xi_{1}\right)\right)^{1 /\left(1-v_{j 1}\right)} \bar{\gamma}^{v_{j 1} /\left(1-v_{j 1}\right)}$, which is a contradiction. Hence (3.14) holds. 
Next, turning to (3.15). If there exists $x_{2} \in P \cap \partial S_{\bar{r}}$ such that $A x_{2} \leq x_{2}$, then for $t \in$ $\left[\xi_{1}, \xi_{2}\right]$, we have

$$
\begin{aligned}
x_{2}(t) & \geq A x_{2}(t) \\
& =\int_{t_{1}}^{t_{m}} G(t, s) f\left(s, x_{2}(s)\right) \Delta s+\Gamma_{2}\left(f\left(t, x_{2}(t)\right)\right) \psi(t)+\Upsilon_{2}\left(f\left(t, x_{2}(t)\right)\right) \phi(t) \\
& \geq \int_{t_{1}}^{t_{m}} G(t, s) f\left(s, x_{2}(s)\right) \Delta s \\
& \geq \int_{\xi_{1}}^{\xi_{2}} G(t, s) c_{j 2}(s)\left[x_{2}(s)\right]^{v_{j 2}} \Delta s \\
& \geq \tau \tau_{2}\left(\xi_{2}-\xi_{1}\right) \bar{\gamma}^{v_{j 2}}\left\|x_{2}\right\|^{v_{j 2}},
\end{aligned}
$$

where $\Gamma_{2}(f(t, x(t))), \Upsilon_{2}(f(t, x(t)))$ are defined by (2.17).

Therefore $\bar{r} \geq \tau \tau_{2}\left(\xi_{2}-\xi_{1}\right) \bar{\gamma}^{v_{j 2}} \bar{r}^{v_{j 2}}$, that is, $\bar{r} \leq\left(\tau \tau_{2}\left(\xi_{2}-\xi_{1}\right)\right)^{-1 /\left(v_{j 2}-1\right)} \bar{\gamma}^{-v_{j 2} /\left(v_{j 2}-1\right)}$, which is a contradiction. Hence (3.15) holds.

It remains to prove

$$
A x \geq x, \quad \forall x \in P \cap \partial S_{1} .
$$

In fact, if there exists $x_{0} \in P \cap \partial S_{1}$ such that $A x_{0} \geq x_{0}$, then for $t \in\left(t_{1}, t_{m}\right) \cap \mathbb{T}$, we have

$$
1=\left\|x_{0}\right\| \leq\left\|A x_{0}\right\| \leq M \sum_{j=1}^{n}\left\|c_{j}\right\|_{L}\left\|x_{0}\right\|^{v_{j}}\left[1+\widetilde{\Gamma} \psi\left(t_{m}\right)+\tilde{\Upsilon} \phi\left(t_{1}\right)\right]
$$

that is,

$$
\sum_{j=1}^{n}\left\|c_{j}\right\|_{L}\left[1+\tilde{\Gamma} \psi\left(t_{m}\right)+\tilde{\Upsilon} \phi\left(t_{1}\right)\right] \geq M^{-1}
$$

which is a contradiction, where $\Gamma_{0}(f(t, x(t))), \Upsilon_{0}(f(t, x(t)))$ are defined by (2.17). Hence (3.18) holds. From Lemma 2.6, (3.14), (3.15) and (3.18) yield that the problem (1.1) has at least two solutions $x_{*}, x_{* *}$ and $x_{*} \in P \cap\left(S_{\bar{r}} \backslash \bar{S}_{1}\right), x_{* *} \in P \cap\left(S_{1} \backslash \bar{S}_{r}\right)$. The proof is complete. 


\section{Example}

Example 4.1. To illustrate how our main results can be used in practice we present an example. Let $\mathbb{T}=\left\{0,1 / 2,1 / 4, \ldots, 1 / 2^{n}, \ldots, 1\right\}$. Take $p(t) \equiv 1, q(t) \equiv 0, \alpha=1, \beta=0, \gamma=1, \delta=$ $0, t_{1}=0, t_{2}=1 / 2, t_{m}=1, a_{2}=1 / 2, b_{2}=1$ in (1.1). Now we consider the following three point boundary value problem

$$
\begin{gathered}
x^{\nabla \Delta}(t)=f(t, x(t)), \quad 0<t<1, \\
x(0)=\frac{1}{2} x\left(\frac{1}{2}\right) \quad x(1)=x\left(\frac{1}{2}\right),
\end{gathered}
$$

where

$$
f(t, x)=\frac{1}{10} t x^{1 / 2}+\frac{1}{20} t^{2} x+\left(t-t^{2}\right) x^{2} .
$$

It is not difficult to see that

$$
c_{1}(t)=\frac{1}{10} t, \quad c_{2}(t)=\frac{1}{20} t^{2}, \quad c_{3}(t)=t-t^{2}, \quad v_{1}=\frac{1}{2}, \quad v_{2}=1, \quad v_{3}=2 .
$$

On the other hand, by calculating we have $\psi(t)=t, \phi(t)=1-t, d=1, d-\sum_{i=2}^{m-1} a_{i} \phi\left(t_{i}\right)=$ $1-1 / 2 \times 1 / 2=3 / 4>0, d-\sum_{i=2}^{m-1} b_{i} \psi\left(t_{i}\right)=1-1 \times 1 / 2=1 / 2>0$,

$$
D=\left|\begin{array}{cc}
-\frac{1}{4} & \frac{3}{4} \\
\frac{1}{2} & -\frac{1}{2}
\end{array}\right|=-\frac{1}{4}<0, \quad G(t, s)=\left\{\begin{array}{l}
s(1-t), \\
t(1-s),
\end{array}\right.
$$

and $M=\max _{t, s \in[0,1]} G(t, s)=1 / 4, \tilde{\Gamma}=7 / 2, \tilde{\Upsilon}=2$.

Let $v_{j 1}=1 / 2, v_{j 2}=2, c_{j 1}(t)=(1 / 10) t, c_{j 2}(t)=t-t^{2}$. Then $v_{j 1}<1, v_{j 2}>1$ and

$$
\inf _{t \in\left[\xi_{1}, \xi_{2}\right]} c_{j 1}(t)=\frac{1}{10} \xi_{1}>0, \quad \inf _{t \in\left[\xi_{1}, \xi_{2}\right]} c_{j 2}(t)=\min \left\{\xi_{1}\left(1-\xi_{1}\right), \xi_{2}\left(1-\xi_{2}\right)\right\}>0, \quad j=1,2,3
$$

It follows that $\left(H_{1}\right)$ and $\left(H_{2}\right)$ hold.

Finally, we prove that

$$
\sum_{j=1}^{n}\left\|c_{j}\right\|_{L}\left[1+\tilde{\Gamma} \psi\left(t_{n}\right)+\tilde{\Upsilon} \phi\left(t_{1}\right)\right]<M^{-1}
$$

In fact, from $\tilde{\Gamma}=7 / 2, \tilde{\Upsilon}=2, \psi(1)=1, \phi(0)=1$, we have $1+\tilde{\Gamma} \psi\left(t_{n}\right)+\tilde{\Upsilon} \phi\left(t_{1}\right)=13 / 2$, and

$$
\sum_{j=1}^{n}\left\|c_{j}\right\|_{L}\left[1+\tilde{\Gamma} \psi\left(t_{n}\right)+\tilde{\Upsilon} \phi\left(t_{1}\right)\right]=\frac{2}{5} \times \frac{13}{2}=\frac{13}{5}<4=M^{-1} .
$$


Therefore, the conditions of Theorem 3.4 hold. Hence problem (4.1) has at least two positive solutions.

Remark 4.2. Example 4.1 implies that there is a large number of functions that satisfy the conditions of Theorem 3.4. In addition, the conditions of Theorem 3.4 are also easy to check.

\section{Acknowledgments}

This work is sponsored by the National Natural Science Foundation of China (10671012) and the Scientific Creative Platform Foundation of Beijing Municipal Commission of Education (PXM2008-014224-067420). The authors thank the referee for his careful reading of the manuscript and useful suggestions.

\section{References}

[1] R. P. Agarwal, M. Bohner, and P. J. Y. Wong, "Sturm-Liouville eigenvalue problems on time scales," Applied Mathematics and Computation, vol. 99, no. 2-3, pp. 153-166, 1999.

[2] R. I. Avery and D. R. Anderson, "Existence of three positive solutions to a second-order boundary value problem on a measure chain," Journal of Computational and Applied Mathematics, vol. 141, no. 1-2, pp. 65-73, 2002.

[3] J. Henderson, "Multiple solutions for $2 m$ th order Sturm-Liouville boundary value problems on a measure chain," Journal of Difference Equations and Applications, vol. 6, no. 4, pp. 417-429, 2000.

[4] D. Anderson, R. Avery, and J. Henderson, "Existence of solutions for a one dimensional $p$-Laplacian on time-scales," Journal of Difference Equations and Applications, vol. 10, no. 10, pp. 889-896, 2004.

[5] M. Bohner and H. Luo, "Singular second-order multipoint dynamic boundary value problems with mixed derivatives," Advances in Difference Equations, vol. 2006, Article ID 54989, 15 pages, 2006.

[6] D. R. Anderson and R. Ma, "Second-order n-point eigenvalue problems on time scales," Advances in Difference Equations, vol. 2006, Article ID 59572, 17 pages, 2006.

[7] A. C. Peterson, Y. N. Raffoul, and C. C. Tisdell, "Three point boundary value problems on time scales," Journal of Difference Equations and Applications, vol. 10, no. 9, pp. 843-849, 2004.

[8] J. Henderson and C. C. Tisdell, "Topological transversality and boundary value problems on time scales," Journal of Mathematical Analysis and Applications, vol. 289, no. 1, pp. 110-125, 2004.

[9] J. Henderson, A. Peterson, and C. C. Tisdell, "On the existence and uniqueness of solutions to boundary value problems on time scales," Advances in Difference Equations, vol. 2004, no. 2, pp. 93-109, 2004.

[10] R. P. Agarwal, M. Bohner, and W.-T. Li, Nonoscillation and Oscillation: Theory for Functional Differential Equations, vol. 267 of Monographs and Textbooks in Pure and Applied Mathematics, Marcel Dekker, New York, NY, USA, 2004.

[11] M. Bohner and A. Peterson, Dynamic Equations on Time Scales: An Introduction with Applications, Birkhäuser, Boston, Mass, USA, 2001.

[12] M. Bohner and A. Peterson, Eds., Advances in Dynamic Equations on Time Scales, Birkhäuser, Boston, Mass, USA, 2003.

[13] D. R. Anderson, "Extension of a second-order multi-point problem to time scales," Dynamic Systems and Applications, vol. 12, no. 3-4, pp. 393-403, 2003.

[14] L. Erbe and A. Peterson, “Green's functions and comparison theorems for differential equations on measure chains," Dynamics of Continuous, Discrete and Impulsive Systems, vol. 6, no. 1, pp. 121-137, 1999.

[15] D. R. Anderson, "Eigenvalue intervals for a two-point boundary value problem on a measure chain," Journal of Computational and Applied Mathematics, vol. 141, no. 1-2, pp. 57-64, 2002.

[16] Z. He and X. Jiang, "Triple positive solutions of boundary value problems for $p$-Laplacian dynamic equations on time scales," Journal of Mathematical Analysis and Applications, vol. 321, no. 2, pp. 911-920, 2006. 
[17] C. J. Chyan and J. Henderson, "Eigenvalue problems for nonlinear differential equations on a measure chain," Journal of Mathematical Analysis and Applications, vol. 245, no. 2, pp. 547-559, 2000.

[18] V. Lakshmikantham, S. Sivasundaram, and B. Kaymakcalan, Dynamic Systems on Measure Chains, vol. 370 of Mathematics and Its Applications, Kluwer Academic Publishers, Dordrecht, The Netherlands, 1996.

[19] S. Hilger, "Analysis on measure chains-a unified approach to continuous and discrete calculus," Results in Mathematics, vol. 18, no. 1-2, pp. 18-56, 1990.

[20] L. Erbe, A. Peterson, and S. H. Saker, "Hille-Kneser-type criteria for second-order dynamic equations on time scales," Advances in Difference Equations, vol. 2006, Article ID 51401, 18 pages, 2006.

[21] R. A. Khan, J. J. Nieto, and V. Otero-Espinar, "Existence and approximation of solution of three-point boundary value problems on time scales," Journal of Difference Equations and Applications, vol. 14, no. 7, pp. 723-736, 2008.

[22] R. P. Agarose, V. Other-Espial, K. Parera, and D. R. Vivre, "Multiple positive solutions in the sense of distributions of singular BAPS on time scales and an application to Eden-Fowler equations," Advances in Difference Equations, vol. 2008, Article ID 796851, 13 pages, 2008.

[23] D.-B. Wang, "Three positive solutions of three-point boundary value problems for $p$-Laplacian dynamic equations on time scales," Nonlinear Analysis: Theory, Methods $\mathcal{E}$ Applications, vol. 68, no. 8, pp. 2172-2180, 2008.

[24] J.-P. Sun, "A new existence theorem for right focal boundary value problems on a measure chain," Applied Mathematics Letters, vol. 18, no. 1, pp. 41-47, 2005.

[25] M. Feng, X. Zhang, and W. Ge, "Positive solutions for a class of boundary value problems on time scales," Computers \& Mathematics with Applications, vol. 54, no. 4, pp. 467-475, 2007.

[26] M.-Q. Feng, X.-G. Li, and W.-G. Ge, "Triple positive solutions of fourth-order four-point boundary value problems of $p$-Laplacian dynamic equations on time scales," Advances in Difference Equations, vol. 2008, Article ID 496078, 9 pages, 2008.

[27] M. Feng, H. Feng, X. Zhang, and W. Ge, "Triple positive solutions for a class of $m$-point dynamic equations on time scales with $p$-Laplacian," Mathematical and Computer Modelling, vol. 48, no. 7-8, pp. 1213-1226, 2008.

[28] X. Xu, Multiple solutions for impulsive singular differential equations boundary value problems, Doctorial thesis, Shandong University, Shandong, China, 2001.

[29] K. Deimling, Nonlinear Functional Analysis, Springer, Berlin, Germany, 1985.

[30] D. J. Guo and V. Lakshmikantham, Nonlinear Problems in Abstract Cones, vol. 5 of Notes and Reports in Mathematics in Science and Engineering, Academic Press, Boston, Mass, USA, 1988. 